\title{
Transverse Schottky noise spectrum for bunches with space charge
}

\author{
O. Boine-Frankenheim and V. Kornilov \\ GSI Helmholtzzentrum für Schwerionenforschung GmbH, Planckstraße 1, 64291 Darmstadt, Germany
}

(Received 24 August 2009; published 20 November 2009)

\begin{abstract}
The effect of space charge and image currents on the transverse Schottky spectrum for bunched beams has been investigated using simulations combined with an analytic model. We found a good agreement between the shifts of synchrotron satellites observed in the simulation noise spectrum and a simplified model for head-tail modes with space charge and image currents.
\end{abstract}

DOI: 10.1103/PhysRevSTAB.12.114201

PACS numbers: 29.20.D-, 29.27.Bd, 52.65.Rr

\section{INTRODUCTION}

Schottky signals play an important role in the diagnostics of ion beams in storage rings and synchrotrons. The Schottky noise spectrum, divided into separate bands, contains information on the incoherent and coherent frequencies in the beam [1]. In many machines the Schottky spectrum is used routinely to measure, e.g., betatron tunes and tune spreads. For high intensity beams at medium energies, such as in the SIS-18 synchrotron at GSI and in the projected FAIR synchrotrons [2], it is important to understand the effect of space charge on the Schottky spectrum. In coasting beams the transverse Schottky bands, centered at the betatron sidebands, are deformed in a characteristic way [3-5]. The deformation provides important information on collective beam properties, such as the space charge tune shift or the Landau damping rate. In bunched beams the Schottky bands split into synchrotron satellites. Because the corresponding bunch modes are shifted by space charge, the position of the satellites also shifts with increasing intensity. In Ref. [6] it was demonstrated that the frequency of longitudinal bunch modes with space charge can be obtained from the longitudinal Schottky spectrum for a "real beam" as well as from the simulation noise spectrum of a "computer beam." In the present study we will present an analysis of the transverse simulation noise spectrum of bunched beams affected by transverse space charge and image currents. The satellites obtained from the spectrum for realistic bunch forms will be compared to head-tail mode frequencies obtained analytically for a simplified "airbag" bunch distribution [7]. Within our simulation we will study moderately strong space charge conditions, meaning that the maximum space charge tune shift in the bunch $\Delta Q_{\max }$ exceeds the synchrotron tune $Q_{s}$ by not more than $\approx 5$. These conditions can presently be met in the GSI heavy-ion synchrotron SIS- 18 . These space charge parameters also correspond to the expected values in the projected synchrotron SIS-100. Head-tail modes in Gaussian bunches for strong space charge $\Delta Q_{\max } / Q_{s}>10$ have been analyzed in Ref. [8]. One of the aims of the present study is to predict from the Schottky noise spectrum the space charge induced fre- quency shifts of head-tail modes starting from the low intensity case. The paper is organized as follows: In Sec. II we briefly describe the theory of Schottky signals from low intensity beams. A simplified analytical model for the effect of space charge and image currents on headtail modes is presented in Sec. III. Simulation results for bunched beams are discussed in Sec. IV.

\section{TRANSVERSE SCHOTTKY NOISE}

The transverse Schottky signal of the beam, detected by a pickup (PU) probe, is given by the transverse beam offset times current,

$$
d(t)=\sum_{j=1}^{N} x_{j}(t) I_{j}(t)
$$

where $x_{j}(t)$ is the offset and $I_{j}(t)$ the current of the $j$ th particle at the position of the PU in the ring (see, e.g., Ref. [1], page 14). The sum extends over all $N$ particles in the beam. The Schottky noise power spectrum as a function of the frequency $f$ is defined as

$$
S\left(Q_{f}\right)=\left|d\left(Q_{f}\right)\right|^{2},
$$

where $d\left(Q_{f}\right)$ is the Fourier transformed PU signal, $Q_{f}=$ $f / f_{0}$ is the tune, and $f_{0}$ the revolution frequency. In coasting beams the transverse Schottky spectrum is centered around the betatron sidebands $Q_{n}=\left(n \pm Q_{0}\right)$, with the bare tune $Q_{0}$ and the harmonic number $n$. In the absence of intensity effects the shape of each band $P_{n}\left(Q_{f}\right)$ reflects the beam's momentum distribution. The width of a band is determined by the chromatic tune spread $\delta Q_{n}=\xi_{\text {eff }} \delta_{\text {rms }}$ with the rms momentum spread $\delta_{\text {rms }}$, the effective chromaticity $\xi_{\text {eff }}=\left[ \pm \xi-\eta_{0}(n \pm Q)\right]$, the chromaticity $\xi$, and the slip factor $\eta_{0}$. For a low intensity bunched beam, the transverse Schottky band splits into equidistant synchrotron satellites,

$$
Q_{n, k}=\left(n \pm Q_{0}\right)+\Delta Q_{k},
$$

where $\Delta Q_{k}=k Q_{s}$ and the synchrotron tune is $Q_{s}$. In the absence of transverse nonlinear field components, the width of each satellite with $k \neq 0$ is determined by the 
synchrotron tune spread,

$$
\delta Q_{k} \approx|k| Q_{s} \frac{\phi_{m}^{2}}{16} \ll \xi_{\mathrm{eff}} \delta_{\mathrm{rms}},
$$

where $\phi_{m}$ is the bunch half-length. For a given sideband the relative amplitude of the synchrotron satellites resembles the momentum distribution in the bunch. Therefore the envelope of the power spectrum duplicates the power spectrum $S\left(Q_{f}\right)$ for the coasting beam with the momentum spread in the bunch center $\delta_{\text {rms }}$ ([1], page 35). For coasting beams, the shape of the Schottky bands can be resolved very well using the simulation noise of particle tracking codes [3]. For bunched beams, the width of the satellites $\delta Q_{k}$ is much smaller than $\xi_{\text {eff }} \delta_{\text {rms }}$. Therefore much longer simulation times are necessary to resolve the shape of the satellites. This is especially a problem if collective effects should be included. Figure 1 shows the simulation noise spectrum obtained from the particle tracking code PATRIC [9] for a low intensity bunch, ignoring collective effects. In our simulations $N_{p}=10^{6}$ macroparticles are initially loaded randomly in 3D phase space to reproduce a matched longitudinal Gaussian bunch distribution in an rf bucket and a transverse Kapchinsky-Vladimirsky (K-V) distribution. The chosen bunch half-length is $\phi_{m}=90^{\circ}$. We limit the simulation time to $T=10 T_{s}$ with $T_{s}=1 /\left(f_{0} Q_{s}\right)$. The tune spread resulting from the finite simulation time is $\delta Q=Q_{s} T_{s} / T$, which is smaller than the expected tune spread in the rf bucket. In order to better display satellites of a broader $k$ range we plot the sum over positive sidebands:

$$
S\left(Q_{f}-Q_{0}\right)=\sum_{n=0}^{n_{\max }} S_{n}\left(Q_{f}-Q_{n}\right)
$$

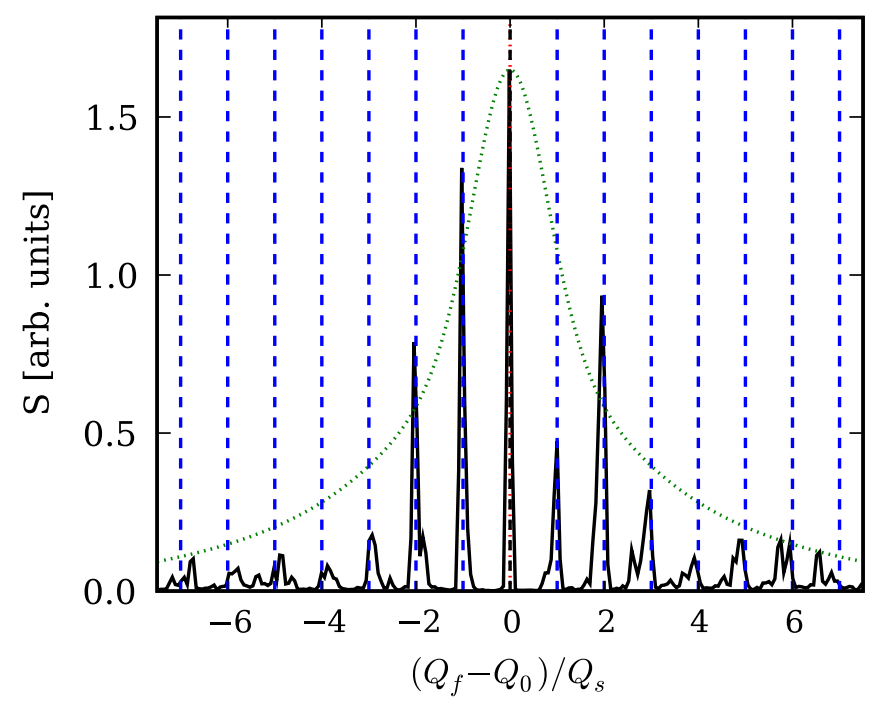

FIG. 1. (Color) Transverse simulation noise spectrum $S_{\Sigma}\left(Q_{f}\right)$ for a matched bunch. The dashed blue lines indicate the synchrotron satellites at $k Q_{s}$. The dotted green curve represents the Schottky spectrum for the equivalent coasting beam.
For the $k$ range chosen in our plots $(|k| \lesssim 8)$, we find that this sum converges for $n_{\max } \approx 10$. In Fig. 1 we compare the simulation noise spectrum $S\left(Q_{f}-Q_{0}\right)$ to the corresponding spectrum for the coasting beam. The synchrotron satellites clearly exhibit a broadening with increasing $|k|$. The shape of each satellite reflects the synchrotron tune distribution in the nonlinear rf bucket. Although the relative amplitudes of the satellites obtained from the simulation noise resemble the coasting beam power spectrum (the dotted green curve in Fig. 1), there are fluctuations that can be explained by the finite number of randomly loaded simulation macroparticles. The experimental observation of transverse Schottky signals from bunched beams is complicated by the coherent longitudinal lines at $f=$ $n f_{0}$ [10]. For coasting beams, we succeeded in taking highly accurate measurements of transverse Schottky bands in the GSI synchrotron SIS-18 [3]. With the available Schottky pickup and without a filter for the coherent lines, we were not able to measure transverse Schottky signals from bunches. Therefore, for the time being, we must rely on studies of noise signals from bunched computer beams. A simplified model for the modification of the spectrum due to collective effects is presented in the next section.

\section{BUNCH MODES WITH SPACE CHARGE AND IMAGE CURRENTS}

For a symmetric beam profile of homogenous density, space charge induces an incoherent tune shift $Q_{0}-\Delta Q_{\mathrm{sc}}$, where

$$
\Delta Q_{\mathrm{sc}}(\phi)=\frac{q I(\phi) R}{4 \pi \varepsilon_{0} c E_{0} \beta_{0}^{3} \gamma_{0}^{2} \epsilon}
$$

is the tune shift, $I(\phi)$ the local beam current, $q$ the particle charge, and $E_{0}$ the energy. The relativistic parameters are $\gamma_{0}$ and $\beta_{0}$, the ring radius is $R$, and the emittance of the rms equivalent $\mathrm{K}-\mathrm{V}$ distribution is $\epsilon$. The corresponding image current induced in the beam pipe, assumed here to be perfectly conducting, causes a coherent tune shift,

$$
\Delta Q_{\mathrm{coh}}=\frac{a^{2}}{b^{2}} \Delta Q_{\mathrm{sc}}
$$

where $a$ is the beam radius and $b$ is the pipe radius. In an intense bunch, the transverse space charge force and the image current will shift the frequencies of head-tail modes and thus the positions of the satellites in the Schottky spectrum as well. The frequency shifts can be obtained analytically for the airbag distribution in a square potential well [7]. Within this model the beam current is constant between the two barriers. There are only the two longitudinal particle velocities $v_{s}= \pm v_{s 0}$. The synchrotron tune is $Q_{s}=\pi v_{s 0} /\left(R \omega_{0} \phi_{b}\right)$, with the full bunch length $\phi_{b}$, the ring radius $R$, and $\omega_{0}=2 \pi f_{0}$. This model makes it simple to analytically calculate the shifted head-tail mode frequencies as 


$$
\begin{aligned}
\Delta Q_{k}= & -\frac{\Delta Q_{\mathrm{sc}}+\Delta Q_{\mathrm{coh}}}{2} \\
& \pm \sqrt{\left(\Delta Q_{\mathrm{sc}}-\Delta Q_{\mathrm{coh}}\right)^{2} / 4+\left(k Q_{s}\right)^{2}},
\end{aligned}
$$

where the + is used for $k>0$. For $k=0$, one obtains $\Delta Q_{k=0}=-\Delta Q_{\text {coh }}$. For weak space charge $\Delta Q_{\text {sc }} \ll Q_{s}$ and weak coherent effects $\Delta Q_{\text {coh }} \ll Q_{s}$, one obtains $\Delta Q_{k}=-\left(\Delta Q_{\mathrm{sc}}+\Delta Q_{\mathrm{coh}}\right) / 2+k Q_{s}$. For strong space charge and weak coherent effects the mode frequencies converge to $\Delta Q_{k}=-\Delta Q_{\text {coh }} / 2$ for $k>0$ and to $\Delta Q_{k}=$ $-\Delta Q_{\mathrm{sc}}-\Delta Q_{\mathrm{coh}} / 2$ for $k<0$. In the extreme case of strong space charge and image charge effects with $\Delta Q_{\mathrm{sc}}=\Delta Q_{\mathrm{coh}}$ positive and negative mode frequencies merge at exactly $\Delta Q_{k}=-\Delta Q_{\mathrm{sc}}$. This shows that coherent effects are less important than space charge for negative mode frequencies. The mode frequencies as a function of $\Delta Q_{\mathrm{sc}}$ are plotted in Fig. 2. If one accounts for space charge only, the eigenfunctions for the square well airbag model are (see Ref. [7], page 10)

$$
x_{ \pm, k}=\left[\cos \left(k \pi \phi / \phi_{b}\right) \mp\left(i \Delta Q_{k} / k Q_{s}\right) \sin \left(k \pi \phi / \phi_{b}\right)\right],
$$

where $X_{+}(\phi, t)=\exp \left(-i Q_{0} \omega_{0} t\right) \exp \left(i \xi \phi / \eta_{0}\right) x_{+}(\phi)$ is the transverse offset for particles with positive $v_{s 0}$. The dipole moment oscillation amplitude for mode $k$ seen by the Schottky pickup is proportional to $\bar{x}_{k}=\left(x_{+, k}+\right.$ $\left.x_{-, k}\right) / 2=\cos \left(k \pi \phi / \phi_{b}\right)$. In addition there is an envelope oscillation amplitude that is not seen by the pickup $\Delta x_{k}=$ $\left(x_{+, k}-x_{-, k}\right)=\left(i \Delta Q_{k} / k Q_{s}\right) \sin \left(k \pi \phi / \phi_{b}\right)$. For strong space charge and for $k<0$, the envelope oscillation dominates. The analytic model does not take into account transverse space charge effects on the envelope oscillations. Therefore, strictly speaking, the $x_{ \pm, k}$ for $k<0$ are

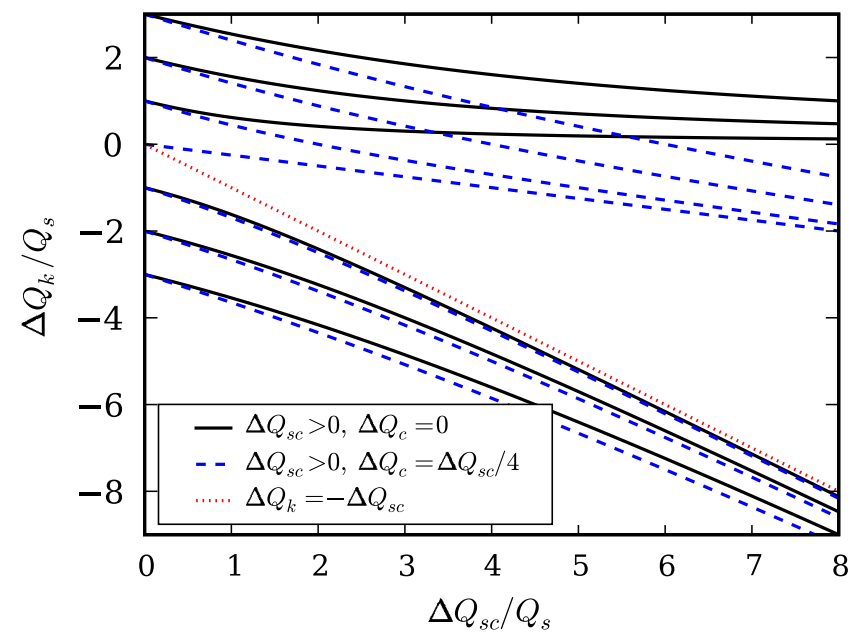

FIG. 2. (Color) Head-tail mode frequencies $\Delta Q_{k}$ as a function of the space charge tune shift $\Delta Q_{\mathrm{sc}}$. The solid black curves are for $\Delta Q_{\text {coh }}=0$. The dashed blue curves are for $\Delta Q_{\text {coh }}=\Delta Q_{\mathrm{sc}} / 4$. The dotted red curve indicates the space charge tune shift $\Delta Q_{k}=-\Delta Q_{\text {sc }}$. not self-consistent solutions and so the $\Delta Q_{k}$ from Eq. (8) are no longer eigenfrequencies for $k<0$ and strong space charge. Another important aspect is that for strong space charge negative modes cannot be excited by a dipolar perturbation. To illustrate this fact we start our PATRIC simulation with a longitudinal airbag distribution and an initial $k=1$ transverse dipole perturbation $\bar{X}(\phi, t=0)=$ $\bar{x}_{1}(\phi)=\hat{\epsilon} \cos \left(\pi \phi / \phi_{b}\right)$. The small initial dipole amplitude is chosen as $\hat{\epsilon} \ll a$. At every location $\phi$ the perturbation is initialized as pure rigid beam offset. This would correspond to a perturbation generated by a fast kicker magnet with a pulse profile that exactly matches $\bar{x}_{1}$ over one turn. The oscillation spectra obtained from the simulation without space charge (solid red curve) and for $\Delta Q_{\mathrm{sc}} / Q_{s}=2$ (solid black curve) are shown in Fig. 3. Without space charge the oscillation spectrum consists of two lines at $\pm Q_{s}$. For moderate space charge the line at $\Delta Q_{-1}$ is strongly reduced and only the positive one at $\Delta Q_{1}$ remains. If we increase space charge above $\Delta Q_{\mathrm{sc}} / Q_{s} \approx 3$ the negative line disappears completely, relatively to the positive one and on this linear scale plot. It is worth mentioning that instead of an initial kick one could also use a periodic signal in order to excite the $\Delta Q_{ \pm 1}$ modes. This would correspond to a transverse beam transfer function (BTF) measurement. Also in this case one observes a decrease of the relative response amplitude for the negative mode with space charge. In summary we showed that with increasing space charge the analytic mode solution for negative $k$ exhibits an envelope oscillation amplitude that is much larger than the dipolar oscillation. The analytic solution for the eigenfrequencies does not account for this envelope oscillation. Therefore the validity of the eigenfrequencies

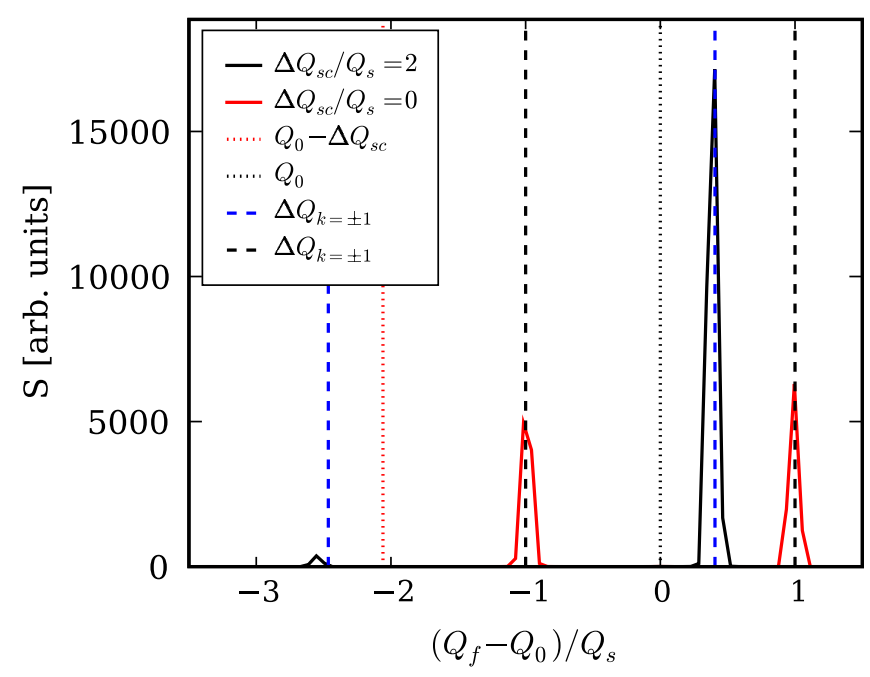

FIG. 3. (Color) Spectrum of the dipole mode $\bar{x}_{1}$ obtained from the PATRIC code for an airbag distribution. The red curve corresponds to $\Delta Q_{\mathrm{sc}} / Q_{s}=0$, the black curve to $\Delta Q_{\mathrm{sc}} / Q_{s}=2$. The dashed blue lines represent $\Delta Q_{ \pm 1}$ for $\Delta Q_{\mathrm{sc}} / Q_{s}=2$. The dashed black lines are at $\pm Q_{s}$. The dotted red line is the tune shifted by space charge. 
for negative $k$ given in Eq. (8) must be questioned. Even for moderately strong space charge negative modes are by far more difficult to excite by a transverse dipolar signal or initial perturbation than positive modes. Because of the analogy between BTF and Schottky signals, one can anticipate that this is a possible reason for the disappearance of the negative Schottky satellites with increasing space charge, observed in the simulations to be discussed in the next section.

\section{SIMULATION OF THE NOISE SPECTRUM WITH SPACE CHARGE}

In this study we use the particle tracking code PATRIC [9] together with a 2.5D space charge solver. The space charge field is solved using an ideally conducting, circular beam pipe as a boundary condition. Usually we choose a ratio of $b / a \approx 4$, so that the coherent tune shift due to image currents is only $\Delta Q_{\text {coh }} \approx \Delta Q_{\text {sc }} / 16$. However, for high intensities the coherent tune shift must be included in the analysis. With the self-consistent space charge solver we limit the simulation time to $T=10 T_{s}$ with $T_{s}=1 /\left(f_{0} Q_{s}\right)$ to enable parameter studies within reasonable times. One should keep in mind that in terms of the resolution needed and the corresponding number of macroparticles the simulation of transverse Schottky spectra from bunched beams is more demanding than for coasting beams. We use the airbag model as described in the previous section together with a transverse $\mathrm{K}-\mathrm{V}$ distribution as a validation example. Figure 4 shows the noise spectrum obtained from the simulation for moderate space charge with $\Delta Q_{\mathrm{sc}} / Q_{s} \approx 2$. The satellites are in very good agreement with the $\Delta Q_{k}$ obtained from Eq. (8). However, for stronger space charge

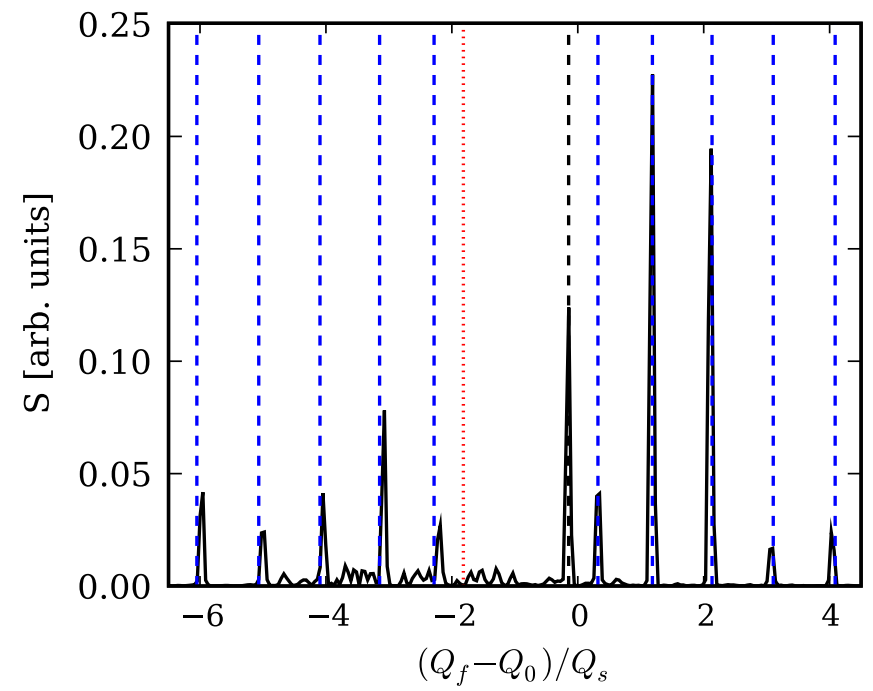

FIG. 4. (Color) Simulation noise spectrum for an airbag distribution in a square well potential and $\Delta Q_{\mathrm{sc}} / Q_{s}=2$. The dashed blue and black $(k=0)$ lines indicate the analytic results from Eq. (8). The dotted red line represents the incoherent space charge tune shift $\Delta Q_{\mathrm{sc}}$.
$\left(\Delta Q_{\text {sc }} / Q_{s} \gtrsim 3\right)$ we find that the satellites with $k<0$ disappear in the simulation noise spectrum (see Fig. 5). Also their position deviates from Eq. (8). Very similar conclusions can be drawn from our simulations for larger coherent shifts with $\Delta Q_{\text {coh }} \approx \Delta Q_{\mathrm{sc}} / 4$. For the airbag model the effect of the image currents in our simulations agrees very well with Eq. (8). For realistic bunch profiles $\Delta Q_{\mathrm{sc}}(\phi)$ varies along the bunch. At the bunch ends $\Delta Q_{\mathrm{sc}}\left(\phi_{m}\right)=0$ holds and $\Delta Q_{\mathrm{sc}}(0)=\Delta Q_{\mathrm{sc}}^{\max }$ in the bunch center. Within our simulation code we studied the noise spectrum from different bunch distributions with the same rms bunch length and particle number. Here we show simulation results for an elliptic bunch distribution in an rf bucket. The bunching factor is chosen as $B_{f}=0.35$. Figure 6 shows the noise spectrum obtained from the simulation for $\Delta Q_{\mathrm{sc}} / Q_{s}=1.5$ together with the mode frequencies predicted from Eq. (8) for $\Delta Q_{\mathrm{sc}}=\Delta Q_{\mathrm{sc}}^{\max }$. The suppression and broadening of the negative satellites can be observed very clearly. Similar to the airbag distribution in a square well potential, one observes that the negative modes disappear in the noise spectrum. A possible reason for this disappearance of the negative Schottky satellites has been given at the end of Sec. III. Moreover, in an rf bucket the lines for $k<0$ can be Landau damped very effectively because they overlap with the incoherent spectrum [8]. The $k=0$ line in Fig. 6 has a relatively small amplitude, whereas the lines for $k>0$ are well pronounced. The position of the $k=1$ satellite matches very well $\Delta Q_{k=1}$ from Eq. (8). With increasing $k$ the observed frequency shifts induced by space charge are underestimated by the airbag model. It is interesting to point out that the relative amplitude of the satellites can be described rather well by

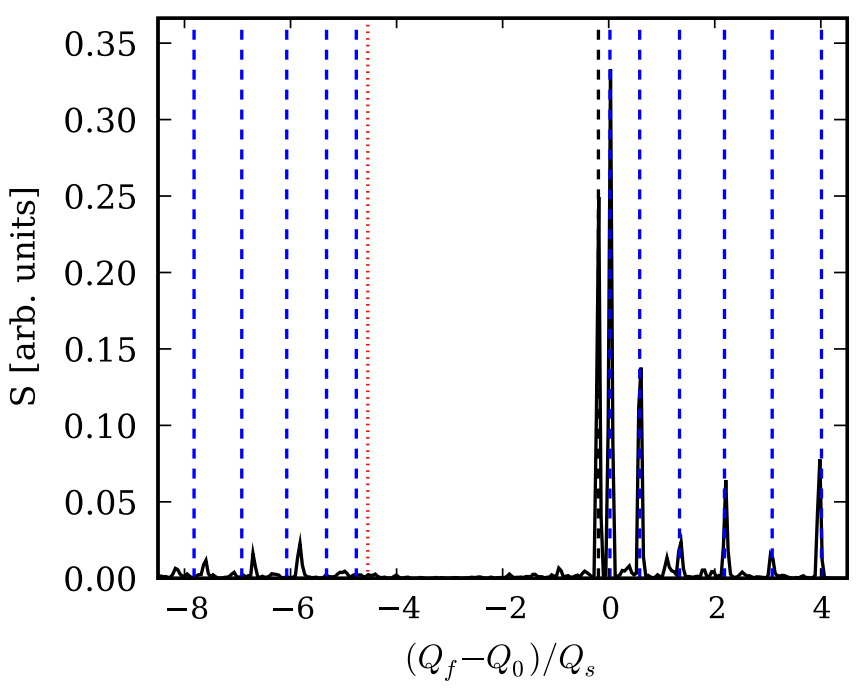

FIG. 5. (Color) Simulation noise spectrum for an airbag distribution in a square well potential and $\Delta Q_{\mathrm{sc}} / Q_{s}=4.5$. The dashed blue and black $(k=0)$ lines indicate the analytic results from Eq. (8). The dotted red line represents the incoherent space charge tune shift $\Delta Q_{\mathrm{sc}}$. 


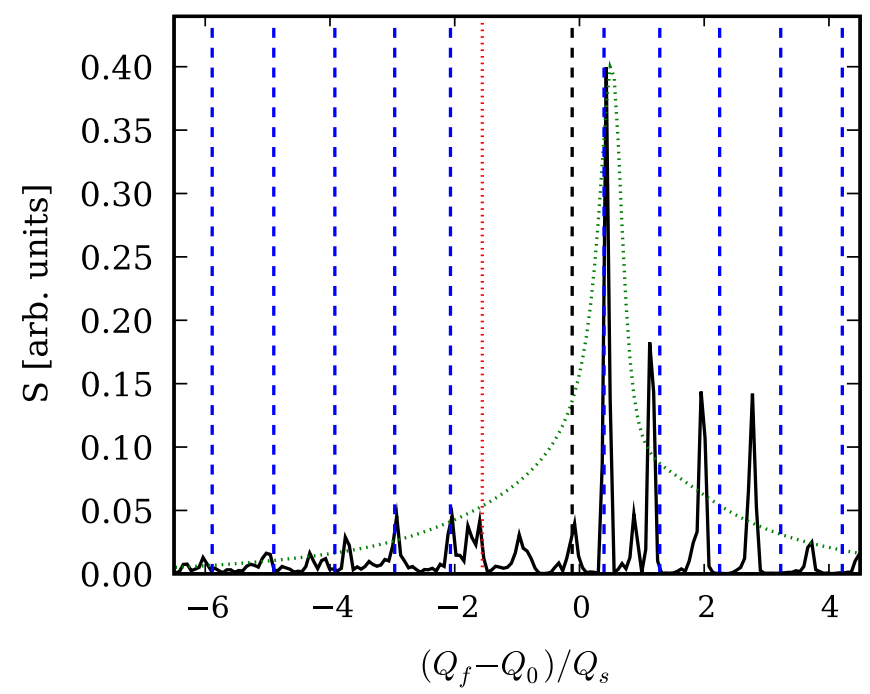

FIG. 6. (Color) Simulation noise spectrum from a parabolic bunch for $\Delta Q_{\mathrm{sc}} / Q_{s}=1.5$ together with $Q_{k}$ from Eq. (8). The dashed blue and black $(k=0)$ lines indicate the analytic results from Eq. (8). The dotted red line represents the maximum incoherent space charge tune shift $\Delta Q_{\mathrm{sc}}$ in the bunch. The dotted green curve represents the Schottky spectrum for the equivalent coasting beam.

the power spectrum for the corresponding coasting beam (dotted green curve in Fig. 6) with a space charge parameter $U_{\text {sc }}=\Delta Q_{\max } / \xi_{\text {eff }}$ (see Ref. [3]). We also observe additional satellites, like the one at $\left(Q_{f}-Q_{0}\right) / Q_{s} \approx-1$ in Fig. 6. These additional lines disappear for stronger space charge. In Fig. 7 one can observe that for stronger space

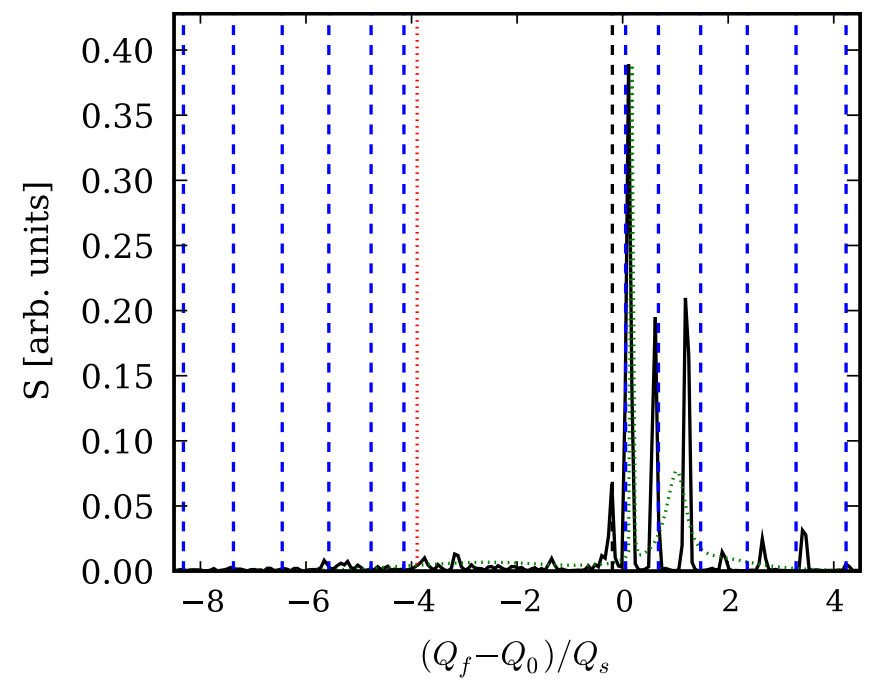

FIG. 7. (Color) Simulation noise spectrum from a parabolic bunch for $\Delta Q_{\mathrm{sc}} / Q_{s}=4.0$ together with $Q_{k}$ from Eq. (8). The dashed blue and black $(k=0)$ lines indicate the analytic results from Eq. (8). The dotted red line represents the maximum incoherent space charge tune shift $\Delta Q_{\mathrm{sc}}$ in the bunch. The dotted green curve represents the Schottky spectrum for the equivalent coasting beam.

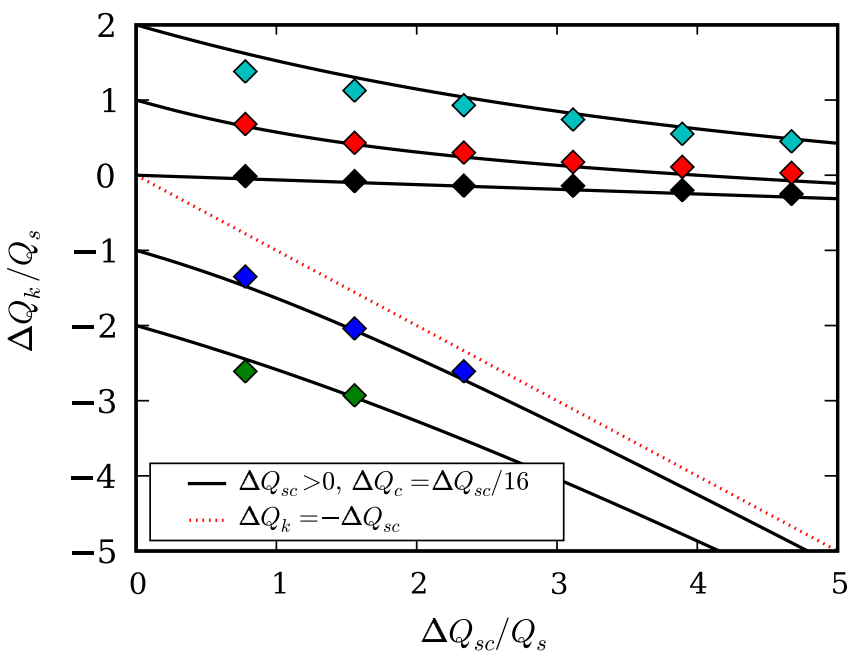

FIG. 8. (Color) Head-tail mode frequencies $\Delta Q_{k}$ as a function of the space charge tune shift $\Delta Q_{s}$. The symbols indicate the mode frequencies obtained from the simulation noise spectrum for an elliptic bunch distribution with $\Delta Q_{\mathrm{sc}}=\Delta Q_{\mathrm{sc}}^{\max }$.

charge (here $\Delta Q_{\mathrm{sc}} / Q_{s} \gtrless 4$ ) the negative satellites completely disappear. The agreement with Eq. (8) for the positive, low $k$ satellites is very good. Again we observe that the relatively low amplitude of the $k=0$ satellite is in agreement with the coasting beam result. In Fig. 8 the frequencies of the satellites in the simulation spectrum for an elliptic bunch distribution as a function of $\Delta Q_{\mathrm{sc}} / Q_{s}$ and for $|k| \leq 2$ are compared with the analytic $Q_{k}$ from the airbag model [Eq. (8)]. One can again observe the good agreement for the positive frequencies with low $k$. The small shift due to image currents is clearly visible for the $k=0$ mode. Also for a Gaussian bunch distribution we obtained a good agreement between the frequencies of the satellites and Eq. (8) with $\Delta Q_{\mathrm{sc}}=\Delta Q_{\mathrm{sc}}^{\max }$. In addition we performed simulation studies using an rms equivalent transverse waterbag distribution instead of a K-V distribution. The obtained Schottky spectra are again very similar. This indicates that the effect of the space charge nonlinearity on the Schottky noise spectrum is weak in the chosen parameter regime.

\section{CONCLUSIONS}

For bunched beams in rf buckets affected by moderately strong transverse space charge and image currents, we compared the shift of the satellites in the simulation noise spectrum with the analytic eigenfrequencies for the airbag distribution in a square potential well. For the airbag distribution and also for more realistic bunch distributions the negative satellites with $k<0$ disappear in the simulation noise spectrum with increasing space charge. A possible reason is that even for moderately strong space charge the negative modes are by far more difficult to excite by a dipolar perturbation than positive modes. The analytic model for the airbag distribution predicts that with increas- 
ing space charge the negative mode frequency shift should asymptotically approach the incoherent space charge tune shift. Therefore the negative Schottky satellites would be the optimum choice to directly measure the incoherent space charge tune shift in bunches. However, we find that the negative Schottky satellites are suppressed for strong space charge and therefore will be difficult to observe experimentally. For positive satellites with $k>0$ and realistic bunch forms, we find a good agreement between the analytic eigenfrequencies and the low $k$ satellites in the simulation spectrum. This suggests to use the positive Schottky satellites (e.g. $k=1$ ) for the measurement of the space charge tune shift in bunches and for the validation of simulation codes. Our results are also important for the analysis of head-tail instabilities with space charge and image currents.

\section{ACKNOWLEDGMENTS}

The authors would like to thank Alexey Burov, FNAL, for fruitful discussions.
[1] S. Chattopadhyay, CERN Report No. 84-11, 1984.

[2] O. Boine-Frankenheim, I. Hofmann, and V. Kornilov, in Proceedings of the 10th European Particle Accelerator Conference, Edinburgh, Scotland, 2006 (EPS-AG, Edinburgh, Scotland, 2006), p. 1882.

[3] O. Boine-Frankenheim, V. Kornilov, and S. Paret, Phys. Rev. ST Accel. Beams 11, 074202 (2008).

[4] A. Burov and V. Lebedev, Phys. Rev. ST Accel. Beams 12, 034201 (2009).

[5] S. Paret, V. Kornilov, O. Boine-Frankenheim, and Th. Weiland (unpublished).

[6] I. Hofmann and G. Kalisch, Phys. Rev. E 53, 2807 (1996).

[7] M. Blaskiewicz, Phys. Rev. ST Accel. Beams 1, 044201 (1998).

[8] A. Burov, Phys. Rev. ST Accel. Beams 12, 044202 (2009).

[9] O. Boine-Frankenheim and V. Kornilov, in Proceedings of the ICAP 2006.

[10] T. Linnecar and W. Scandale, in Proceedings of the PAC 1981, p. 2147. 